\title{
Nephrologisch-gefäßchirurgisches Kolloquium Neue Wege gehen
}

Am 28.11.2015 fand das 6. nephrologisch-gefäßchirugische Kolloquium im St. Franziskus-Hospital in Münster statt. Der Fokus der Vorträge lag auf dem Thema „Interdisziplinäre Therapie des Nierenversagens auf der Intensivstation“. Das 7. nephrologisch-gefäßchirurgische Kolloquium wird im Herbst 2017 an gewohnter Stelle durchgeführt werden.

Die Vortragsthemen auf dem 6. nephrologisch-gefäßchirugischen Kolloquium im St. Franziskus-Hospital in Münster waren:

- Pflegerische Aspekte der NET aus Sicht der Intensiv-Fachpflege und der nephrologischen Fachpflege

- Peritonealdialyse als Behandlungsoption auf der Intensivstation?

- Was sollte der Nephrologe zu den neuen Möglichkeiten der maschinellen Herz-Lungen-Unterstützung wissen?

- Wie ist der Stellenwert der SLEDD beim akuten und chronischen Nierenversagen auf der Intensivstation?

- Wie funktioniert eine interventionelle Shuntanlage und für wen ist sie geeignet?
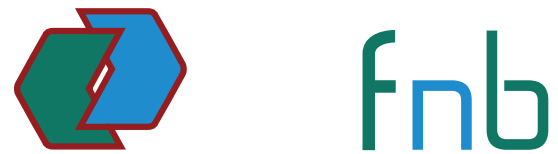

Fachverband nephrologischer Berufsgruppen

Geschäftsstelle fnb, Uschi Gaspar

In den Beunen 6, 65479 Raunheim,

Tel.: 06142/408549, Fax: 06142/408551

E-Mail: uschi.gaspar@nephro-fachverband.de,

www.nephro-fachverband.de

Vorstand des fnb:

- Michael Reichardt (1. Vorsitzender)

- Jürgen Berner (2. Vorsitzender)

- Ina Wiegard-Szramek (Schatzmeisterin)

- Marvin Breckling (Schriftführer)

\section{Die interventionelle Shuntanlage} weckte großes Interesse

Unter der Auswahl hochaktueller Themen weckte insbesondere der letzte Vortrag von Oberarzt Dr. Arne Schwindt, endovaskulärer Chirurg des St. Franziskus-Hospitals Münster, das Interesse der Anwesenden: Die beschriebene Form der Shuntanlage ist neu und das St. Franziskus-Hospital in Münster eines der wenigen Zentren in Europa, welches dieses Verfahren durchführt.

Bei diesem innovativen Verfahren wird der Shunt ohne chirurgischen Schnitt erstellt. Hierzu sind 2 Punktionen und das Einführen kleiner Gefäßkatheter in Arterie und Vene des zukünftigen Shuntarmes notwendig. Dies geschieht in örtlicher Betäubung. Die beiden Katheter sind mit Magneten ausgestattet, welche die beiden Gefäße aneinander pressen können. Durch einen kurzen Hochfrequenzimpuls werden Arterie und Vene in einem zweiten Schritt miteinander verschmolzen, wodurch unmittelbar ein arteriovenöser Shunt entsteht.

Der Eingriff ist komplett minimalinvasiv. Weitere Vorteile sind die Vermeidung von chirurgischen Narben an Haut und Unterhaut und der Wegfall von Klemmungen an den Gefäßen, die bei der herkömmlichen, invasiven Shuntanlage notwendig sind. Diese Klemmungen können im Langzeitverlauf zur Entstehung von Stenosen führen, die dann oft behandelt werden müssen.

Es liegen bereits positive klinische Erfahrungen an über 90 Patienten in Kanada und Südamerika vor, die zur europäischen Zulassung des Kathetersystems geführt haben. Das Fallbeispiel einer erfolgreichen Shuntanlage am St. Franziskus-Hospital mit anschließender Einleitung der Dialysebehandlung verdeutlichte die theoretischen Ausführungen von Schwindt.

\section{Nächste Veranstaltung \\ im Herbst 2017}

Im Herbst 2017 wird das 7. nephrologisch-gefäßchirurgische Kolloquium am St. Franziskus-Hospital in Münster stattfinden. Der Gefäßzugang wird 2017 wieder im Fokus der Aufmerksamkeit stehen. Sehr spannend wird eine Betrachtung und Bewertung der Entwicklung des beschriebenen Shuntverfahrens sein. Weitere Themen werden Diagnostik, Behandlung und Verlaufsformen von Infektionen sowie Infektions-Prophylaxe-Möglichkeiten sein. Praktische pflegerische Vorträge zu Themen wie Nadelfixierung und Fotodokumentation werden für alle Fachdisziplinen rund um den Dialysepatienten von hohem Interesse sein.

Timo Meusel, Münster

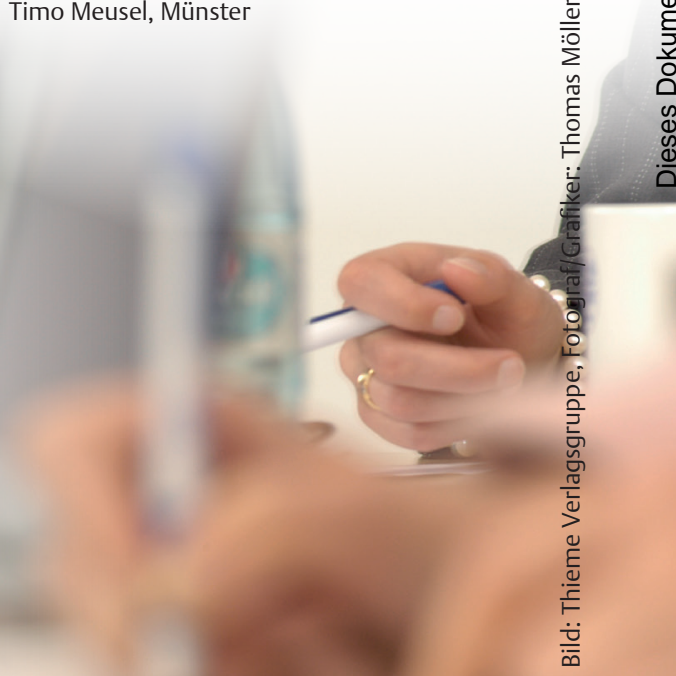

